\title{
OPTIMIZATION OF COMPLEX SYSTEM RELIABILITY USING HYBRID GREY WOLF OPTIMIZER
}

\author{
Ganga Negi1 , Anuj Kumar², Sangeeta Pant², Mangey Ram,4 \\ ${ }^{1}$ Department of Mathematics, Graphic Era Deemed to be University, Dehradun, India \\ ${ }^{2}$ Department of Mathematics, University of Petroleum and Energy Studies, Dehradun, \\ India \\ ${ }^{3}$ Department of Mathematics, Computer Science and Engineering, Graphic Era, \\ Dehradun, India \\ ${ }^{4}$ Institute of Advanced Manufacturing Technologies, Peter the Great St., Petersburg \\ Polytechnic University, Saint Petersburg, Russia
}

Received: 30 March 2021;

Accepted: 20 July 2021;

Available online: 18 August 2021.

Original research paper Abstract: Reliability allocation to increase the total reliability has become a successful way to increase the efficiency of the complex industrial system designs. A lot of research in the past have tackled this problem to a great extent. This is evident from the different techniques developed so far to achieve the target. Metaheuristics like simulated annealing, Tabu search (TS), Particle Swarm Optimization (PSO), Cuckoo Search Optimization (CS), Genetic Algorithm (GA), Grey wolf optimization technique (GWO) etc. have been used in the recent years. This paper proposes a framework for implementing hybrid PSO-GWO algorithm (HPSOGWO) for solving reliability allocation and optimization problems of Complex bridge system and Life support system in space capsule. The supremacy/competitiveness of the proposed framework are demonstrated from the numerical experiments. Comparison of the results obtained by HPSOGWO with previously used algorithms named PSO and GWO shows that in one problem named the Complex bridge system, the HPSOGWO uses lesser number of function evaluations as compared to PSO and GWO. Hence, the overall solutions obtained by HPSOGWO are not only comparable to the previously obtained results by some of the other well-known optimization methods, but also better than that.

Keywords: Cost function, Metaheuristics, Reliability allocation problems, Particle Swarm Optimization (PSO), Grey Wolf Optimizer (GWO), Hybrid PSOGWO algorithm (HPSOGWO).

* Corresponding author.

E-mail addresses: gnegiji@gmail.com (G. Negi), anuj4march@gmail.com (A. Kumar), pant.sangeet@gmail.com (S. Pant), mangeyram@gmail.com (M. Ram) 


\section{Introduction}

The present-day real-world problems of engineering have reached to an advanced level that have motivated the researchers to find ways to increase the efficiency of complex systems. Reliability being the main criteria for this task and the attention of the researchers is diverted towards allocating reliability to the complex systems and the components. Nowadays there has been lot of research in the field of reliability optimization considering its wide applications in real life and industry (Atiqullah \& Rao, 1993; Pham et al., 1995; Eiben \& Schippers, 1998; Kishor et al., 2009; Jayabarathi et al., 2016;). Such improvisations increase the efficiency and give better results for stochastic nonlinear optimization problems (Ramírez-Rosado \& Bernal-Agustín, 2001). The constraints of weight, budget, volume, can be appropriately set, in reliability allocation problem (RAP) to optimize reliability of the system (Kishor et al., 2007; Pant et al., 2015). Due to the immense applications, RAP problems have attracted the attention of many researchers to explore this technology (Mohan \& Shanker, 1987; Majety et al., 1999; Pant \& Singh, 2011; Kumar et al., 2016). Basically, reliability optimization problems can be classified into three categories depending upon the decision variables involved. These are (i) reliability allocation (Li et al., 2008; Mirjalili et al., 2016; Pant et al., 2017; Kumar et al., 2019a, 2019b;) (ii) redundancy allocation (Atiqullah \& Rao, 1993; Misra \& Sharma, 1991a, 1991b; Yang \& Deb, 2009) and (iii) reliability- redundancy allocation (Sakawa, 1978; Coelho, 2009; Deep \& Deepti, 2009). Going by the concept of mathematical programming reliability allocation is a continuous nonlinear programming problem (NLP). Redundancy allocation is a pure integer nonlinear programming problem (INLP) for nonlinear polynomial hard problems. Reliability-redundancy allocation is covered under mixed integer nonlinear programming problem (MINLP) for solving problems of nonconvex nature and combinatorial search space.

Last few decades have witnessed much research in the field of reliability allocation problem (RAP) and reliability optimization by researchers to solve single objective and multiple objective optimization problem. Basically, the solutions techniques used so far to solve RAP and optimization problems are approximation, exact, heuristic and metaheuristic methods. Among these are exact solution techniques for RAP like the cutting plane algorithm was proposed by Majety et al. (1999) with discrete-cost reliability data for components and other such techniques by Hikita et al. $(1986,1992)$. Random search algorithm for RAP presented by Mohan \& Shankar (1987) for complex system reliability optimization. Three levels decomposition approach the Khun Tucker multiplier method for RAP was given by Salazar et al. (2006). Among the metaheuristic techniques for RAP Ant colony technique applied by Shelokar et al. (2002); NSGA 2 by Kishore et al. (2007, 2009); PSO by Pant et al. (2011); CSA by Kumar et al. (2016). These optimization techniques yield solutions for problems of convex nature and monotonicity.

\section{Literature review}

In order to solve complex reliability allocations problems and reliability redundancy allocation problems which are nonlinear optimization problems of nonconvex nature and combinatorial search spaces more advanced algorithms called the metaheuristics have been formulated. These require lot of computational effort to find optimal solutions. As proposed by Wolpert \& Macready (1997) that one type of optimization algorithm is not enough for all optimization problems. So, some researchers are constantly working on developing different types of nature inspired 
meta-heuristics technique. Some of them recently developed are Evolutionary algorithm (EA) (Ramírez-Rosado \& Bernal-Agustín, 2001), Ant colony optimization (ACO), (Zha et al., 2007; Dorigo \& Gambardella, 1997) Particle Swarm Optimization Algorithm (PSO) (Eberhart \& Kennedy, 1995; Kennedy \& Eberhart, 1997; Hu \& Eberhart, 2002, Pant \& Singh, 2011) Grey wolf optimization technique (GWO) (Mirjalili et al. 2014; Fouad et al., 2015; Jayabarathi et al., 2016; Mosavi et al., 2016; Kumar et al., 2017; Kumar et al., 2019a, 2019b; Pant et al., 2019;), Flower pollination Algorithm (Pant et al., 2017) and Cuckoo search algorithm (CSA) (Yang \& Deb, 2009). The detailed reviews of reliability optimization especially GWO, PSO optimization techniques are given by Kuo and Prasad (2000); Negi et al. (2020); Padhye et al. (2009); Uniyal et. al. (2020).

These previous researches have led to the development of some of the recent researches in the field of metaheuristic algorithms and hybrid metaheuristic algorithms and their applications.

Hassan \& Rashid (2021) proposed a new evolutionary clustering algorithm (ECA) based on social class ranking and meta-heuristic algorithms for stochastically analysing heterogeneous and multifeatured datasets. Rahman \& Rashid (2020) presented the idea of Learner performance-based behavior algorithm LPB inspired from the process of accepting graduated learners from high school in different departments at university and has a greater ability to deal with the large optimization problems. A collaborative working approach to path finding was introduced by Shamsaldin et al. (2019) in the form of Donkey and smuggler optimization algorithm to solve different problems such as TSP, packet routing, and ambulance routing. Abdullah \& Ahmed (2019) proposed Fitness Dependent Optimizer inspired by the Bee Swarming Reproductive Process which uses the problem fitness function value to produce weights for guiding during the exploration and exploitation phases. Some of the modified and hybrid algorithms have also been in the recent years to solve many real-world engineering problems. A new K-means grey wolf algorithm was developed by Mohammed et al. (2021) to enhance the limitations of the wolves' searching process of attacking gray wolves. A novel hybrid WOA-GWO presented by Mohammed \& Rashid (2020) by embedding the hunting mechanism of GWO into the WOA exploitation phase with the enhanced exploration for global numerical optimization and to solve the pressure vessel design problem. Mohammed et al. (2019) introduced a systematic and meta-analysis survey of Whale Optimization Algorithm modifying and hybridizing WOA algorithm with BAT algorithm in order to avoid local stagnation as well as increase the rate of convergence to achieve the global optimum solution. Ibrahim et al. (2020) presented a hybrid meta-heuristic algorithm of Shuffled Frog Leaping Algorithm and Genetic Algorithm (SFGA), an energy efficient service composition mechanism consuming minimum cost, response time and energy in a mobile cloud environment as compared to other algorithms. Muhammed et al. (2020) proposed an Improved Fitness-Dependent Optimizer Algorithm IFDOA by first doing the randomization and then minimization of the weight fitness values using it in aperiodic antenna array designs. To forecast students' outcomes by improving the faculty and students' learning experiences Rashid et al. (2019) presented a hybrid system a multi hidden recurrent neural network with a modified grey wolf optimizer. Mukherjee et al. (2021) presented the idea of a multi-objective antlion optimizer for the ring tree problem with secondary sub-depots (MORTPSSD), to overcome the problems of telecommunication and logistics networks by minimizing the circuits' total routing cost. In addition to the above optimizer for secondary depots Mukherjee et al. (2021) introduced a modified discrete antlion optimizer for the ring star problem 
Negi et al./Decis. Mak. Appl. Manag. Eng. 4 (2) (2021) 241-256

(RSPSSD) which overcomes the challenge of minimizing the cost by selecting the suitable primary and secondary subdepots.

In this paper, we present use of hybrid optimization technique called hybrid PSOGWO for reliability allocation problems. The idea is to apply the HPSOGWO algorithm to minimize the cost of the Complex Bridge system and Life support system in a space capsule resulting in better performance in terms of number of cost function evaluations and number of search agents used in PSO and GWO algorithms individually. Both PSO and GWO are population-based swarm intelligence (SI) techniques. Both involve less and only suitable parameters, which have easy application and execution together with optimum convergence to the global solution. That's why it yields better results than other metaheuristics.

Section 2 consists of detailed explanation of the Particle swarm optimization technique. Section 3 involves description of the grey wolf optimization and section 4 gives an overview of hybrid algorithms and describes Hybrid PSO GWO algorithm (HPSOGWO). The formulation of the mathematical models for the proposed problems have been presented in section 5. Section 6 analyses the results of the optimization techniques used. Section 7 presents the conclusion and scope for further results.

\section{Particle Swarm optimization technique (PSO)}

Particle swarm optimization (PSO) simulates the social behaviour of birds of a flock. (Kennedy \& Eberhart, 1997; Hikita et al., 1992; Pant \& Singh, 2011; Abd-Elazim \& Ali, 2015). It is a population-based optimization technique. The randomly generated population of the initial swarm or the particles and their random velocities start the initial process of algorithm. Pbest represents the personal best position of each particle whereas $G_{\text {best }}$ denotes the particle with the best value of fitness and hence called the global best particle. In the $D$-dimensional search space $X_{i}=\left(x_{i}^{1}, x_{i}^{2}, \ldots \ldots \ldots \ldots x_{i}^{D}\right)^{\mathrm{r}}$ and $V_{i}=\left(v_{i}^{1}, v_{i}^{2}, \ldots \ldots \ldots \ldots v_{i}^{D}\right)^{\mathrm{r}}$ denote the position and velocity of the $i^{\text {th }}$ particle whereas the previous best position of the $i^{t h}$ particle is denoted by $P_{i}=\left(p_{i}^{1}, p, \ldots \ldots \ldots \ldots p_{i}^{D}\right)^{\mathrm{r}}$. According to the fitness the best particle is denoted by $P_{g}=\left(p_{g}^{1}, p_{g}^{2}, \ldots \ldots \ldots \ldots p_{g}^{D}\right)^{\mathrm{r}}$ which is the global best particle.

The change in the position and velocities are expressed by the equations: (Kennedy \& Eberhart,1997; Hikita et al., 1992; Abd-Elazim \& Ali, 2015)

$V_{i d}^{k+1}=\left\{\begin{array}{c}V_{\max }, \quad \text { if } V_{i d}^{k+1}>V_{\max } \\ -V_{\max }, \text { if } V_{i d}^{k+1}<-V_{\max } \\ V_{i d}^{k+1}, \quad \text { otherwise }\end{array}\right.$

$x_{i d}^{k+1}(t+1)=x_{i d}^{k}(t)+v_{i d}^{k+1}(t+1)$

Here $i=1,2,3 \ldots . . N ; N=$ swarm size, $k=$ iteration number, $d=1,2,3 \ldots . . w=$ inertia weight, (for controlling the momentum of the particle by weighing the contribution of the previous velocity), $c_{1}$ and $c_{2}$ are the positive acceleration coefficients; $r_{1}$ and $r_{2}$ are the random numbers between 0 and 1 . The variations in $c_{1}$ and $c_{2}$ with the time are represented by following equations respectively

$c_{1}(t)=c_{1 i}+\left(c_{1 f}-c_{1 i}\right) * I T E R / I T E R M A X$

$c_{2}(t)=c_{2 i}+\left(c_{2 f}-c_{2 i}\right) * I T E R / I T E R M A X$

Here, initially value of $c_{1}$ is kept large and value of $c_{2}$ is kept small to ensure enough exploration of the search space to avoid local stagnation. This will lead to the global best solution in the long run. Then, small value of $c_{1}$ and large value $c_{2}$ leads to the 
population best that is the global optimum solution. The maximum velocity and position that the particle can attain in each dimension are given by the equation as follows:

$V_{i d}^{k+1}=\left\{\begin{array}{c}V_{\max }, \quad \text { if } V_{i d}^{k+1}>V_{\max } \\ -V_{\max }, \text { if } V_{\text {id }}^{k+1}<-V_{\max } \\ V_{i d}^{k+1}, \quad \text { otherwise }\end{array}\right.$

$x_{i d}^{k+1}=\left\{\begin{array}{cl}X_{\max }, & \text { if } x_{i d}^{k+1}>X_{\max } \\ X_{\min }, & \text { if } x_{i d}^{k+1}<X_{\max } \\ x_{i d}^{k+1}, & \text { otherwiswe }\end{array}\right.$

\section{Grey Wolf optimization}

\subsection{The guiding factor for the algorithm}

GWO presented by Mirjalili et al. (2014) is an optimization technique which is based on the hierarchical behaviour and social intelligence of the wolves. The entire mechanism of hunting is carried out by the four categories of wolves together. Each category of wolf has a particular role. Alpha, the leader category takes the decisions regarding the whole process and the others follow them. Thus, GWO algorithm based on this very principle is used to find the global optimum solution. The next in the hierarchy are the beta followed by delta and omega. These four initially become the candidates of solution and which are improved in the gradually in further iterations.

\subsection{Mathematical Model formulation of the GWO Algorithm}

The model comprises of:

- $\quad$ Surveying

- $\quad$ Surrounding

- Attacking

The whole process of change of position of the attacking wolves is shown by the following equations constructed to carry out the simulation are as follows. (Mirjalili et al. 2014)

$D=\left|C \cdot X_{p}(t)-X(t)\right|$

$X(t+1)=X(t)-A \cdot D$

Note that in the equations, vectors are used so they are applicable to any number of dimensions. $X(t), X(t+1)$ show the present and the new locations of the wolf. The location of the prey is represented by the vector D. Following equations are useful to calculate the value of $\mathrm{A}$ and $\mathrm{C}$ :

$A=2 a \cdot r_{1}-a$

$C=2 \cdot \mathrm{r}_{2}$

where, $r_{1}$ and $r_{2}$ are random vectors in the interval $[0,1]$. The components of vector a are linearly decreased from 2 to 0 over the course of iterations. The value of A ranges from -2 to 2 as there are random variables in the expression. It is supposed that, alpha, beta and delta are the three best solutions in GWO as they have good idea of the 
location because they are the strongest in the entire population. So, the other wolf should try to update their position as follows.

$X(t+1)=\frac{1}{3} X_{1}+\frac{1}{3} X_{2}+\frac{1}{3} X_{3}$

where, $X_{1}, X_{2}, X_{3}$ are calculated with the equations:

$X_{1}=X_{\alpha}(t)-A_{1} \cdot D_{\alpha}$

$X_{2}=X_{\beta}(t)-A_{2} \cdot D_{\beta}$

$X_{3}=X_{\delta}(t)-A_{3} \cdot D_{\delta}$

Here, $D_{\alpha}, D_{\beta}, D_{\delta}$ are calculated as follows:

$D_{\alpha}=\left|C_{1} \cdot X_{\alpha}-X\right|$

$D_{\beta}=\left|C_{2} \cdot X_{\beta}-X\right|$

$D_{\delta}=\left|C_{3} \cdot X_{\delta}-X\right|$

Pseudo code of GWO is given in Figure 1 (Mirjalili et al., 2014).

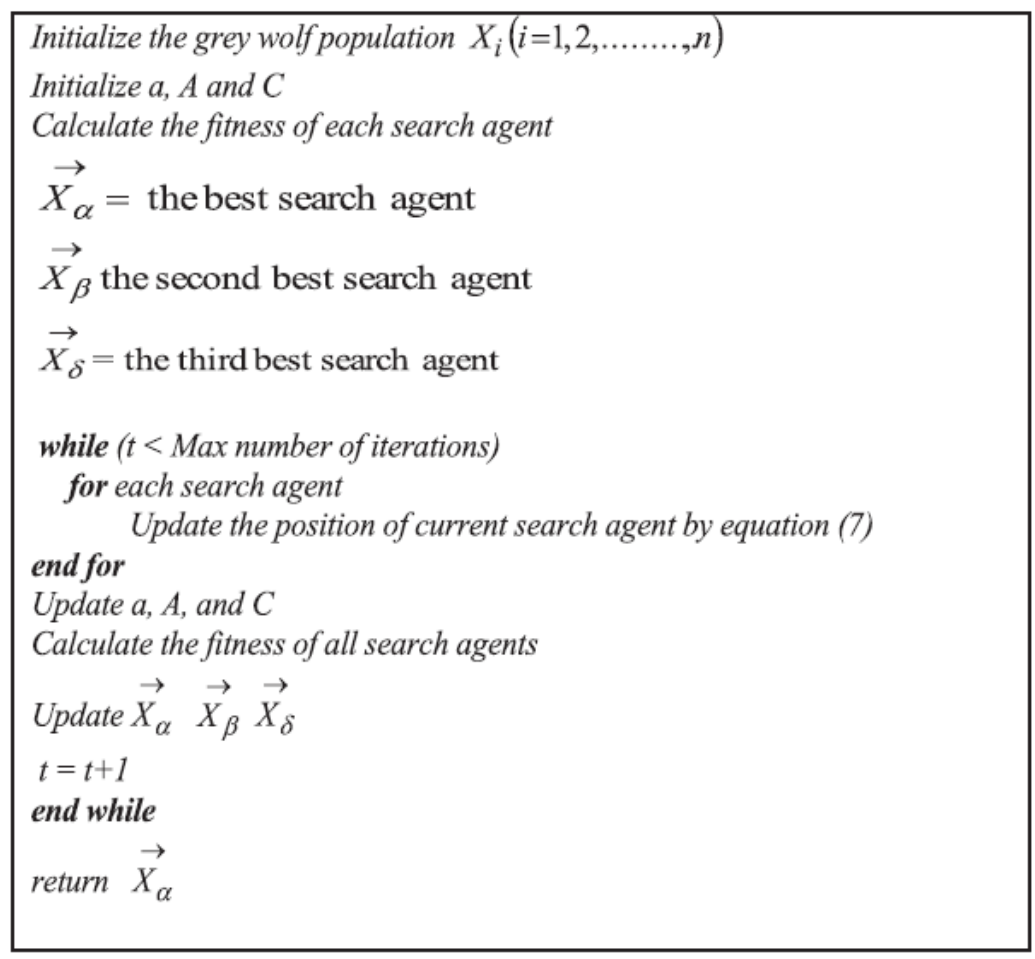

Figure1. Pseudo Code of the GWO Algorithm

\subsection{Balancing of the effective hunting mechanisms:}

It is very essential to do enough surveying before attacking the prey so as to make the hunting mechanism a success. The leading wolves decide and the wolves following the leaders can then take the appropriate positions to encircle the prey. For this parameter a has to be chosen so as to get the suitable value of A correspondingly which should be between -1 and 1 . Exploration is followed by exploitation. To stimulate proper exploitation of the available conditions, the parameter setting requires IAI $<1$. The success of the exploitation is dependent to a great extent on rigorous and balanced exploration so that the result is not stagnated and unrefined. GWO efficiently helps in achieving this. 


\section{Hybrid algorithms}

To use the best qualities of some metaheuristics together the attention of the researchers has now been attracted by hybrid of two metaheuristic together solve s the purpose of reaching the global best solution with results much better than the individual metaheuristics as such in terms of quality, time, better convergence rate. Generally, the phenomena of exploration and exploitation (Eiben \& Schippers, 1998) are regarded as if they cannot go hand in hand and one disturbs the progress of the other. But a balance of these two phenomena actually, leads to the global optimum which is the best solution in terms of avoiding local stagnation, appropriate convergence rate and better result. In using a hybrid of two metaheuristic techniques, they can be used at two levels. One could be low level and other could be high level. Along with this the hybridization could be done in two ways. One is as relay that is one after the other and the other method is coevolutionary which means the techniques hybridized are run parallelly and not one after the other. Since the two different techniques are used in generating the final solution of the problem so it is said that a hybrid is a mixed kind of a technique. Now here the challenge lies in choosing the appropriate level to which the techniques are used. As well as, the suitable method used either relay of parallel. A slight difference in the choices made could lead to the global best solution or no better solution at all. Some of the hybrid techniques used successfully so far by the researchers are GWO-ACO (Ab Rashid, 2017), GWO-GA (Singh \& Singh, 2017), GWO-ANN (Tawhid \& Ali, 2017) and PSO-ACO (Holden \& Freitas, 2008). To enable the process of exploitation, hybrids of PSO have been developed by many researchers. Mirjalili \& Hashmi, (2010) proposed hybrid PSO with Gravitational search algorithm (GSA) that is PSO-GSA to combine the advantages of the PSO with those of the GSA for better performance to escape from local convergence. HPSO by Ahmed et al., (2013) aims at using Particle swarm optimization with (PSO) with Genetic algorithm (GA) mutation technique which give much better result than the PSO. Abd-Elazim \& Ali, (2015) introduced a hybrid of bacterial foraging optimization algorithm (BFOA) and PSO called Bacterial swarm optimization (BSO) which has proved to be testifier in tuning with SVC. In order to avoid local stagnation and obtain better quality in terms of global best and stability factor GWO has been hybridized with many other optimization techniques.

\subsection{Hybrid PSO-GWO algorithm}

It is clear that to improve the convergence behaviour of the metaheuristic technique researchers have started developing hybrids of some of the meta heuristics as mentioned earlier. One of those is hybrid PSO GWO technique (HPSOGWO) (Singh \& Singh, 2017). The advantage of Hybridization of the PSO and GWO technique is that with GWO, the exploration technique is improved as the wolves do enough exploration of the search space. Whereas, PSO helps in improving the exploitation so that the convergence to the solution can be achieved timely as well as to the global optimum. Proper exploitation and exploration with a balance is maintained. This ultimately complements and strengthens the performance of both the techniques taken together avoiding the influence of the shortcomings in terms of local stagnation or suitable convergence rate. The modifications done in the related equations are shown by the use of an inertia weight constant.

For this, the positions of the search agents are to be improved first so that the searching and the exploring process can be bettered. This will automatically control the exploitation and exploration phenomena as a whole. 
Introduction of the inertia constant to control the surveying and attacking processes of the wolves can be expressed as follows. (Singh \& Singh, 2017).

$D_{\alpha}=\left|C_{1} \cdot X_{\alpha}-w * X\right|$

$D_{\beta}=\left|C_{2} \cdot X_{\beta}-w * X\right|$

$$
D_{\delta}=\left|C_{3} \cdot X_{\delta}-w * X\right|
$$

To enhance the exploitation capacities of the PSO the velocity and upgraded locations of the search agents are expressed by the equations as follows:

$$
\begin{gathered}
V_{i}^{k+1}=w *\left\{V_{i}^{k}+c_{1} r_{1}\left(x_{1}-x_{i}^{k}\right)+c_{2} r_{2}\left(x_{2}-x_{i}^{k}\right)+c_{3} r_{3}\left(x_{3}-x_{i}^{k}\right)\right\} \\
x_{i}^{k+1}=x_{i}^{k}+v_{i}^{k+1}
\end{gathered}
$$

Pseudo Code of the HPSOGWO Algorithm is given in Figure 2. (Singh \& Singh, 2017).

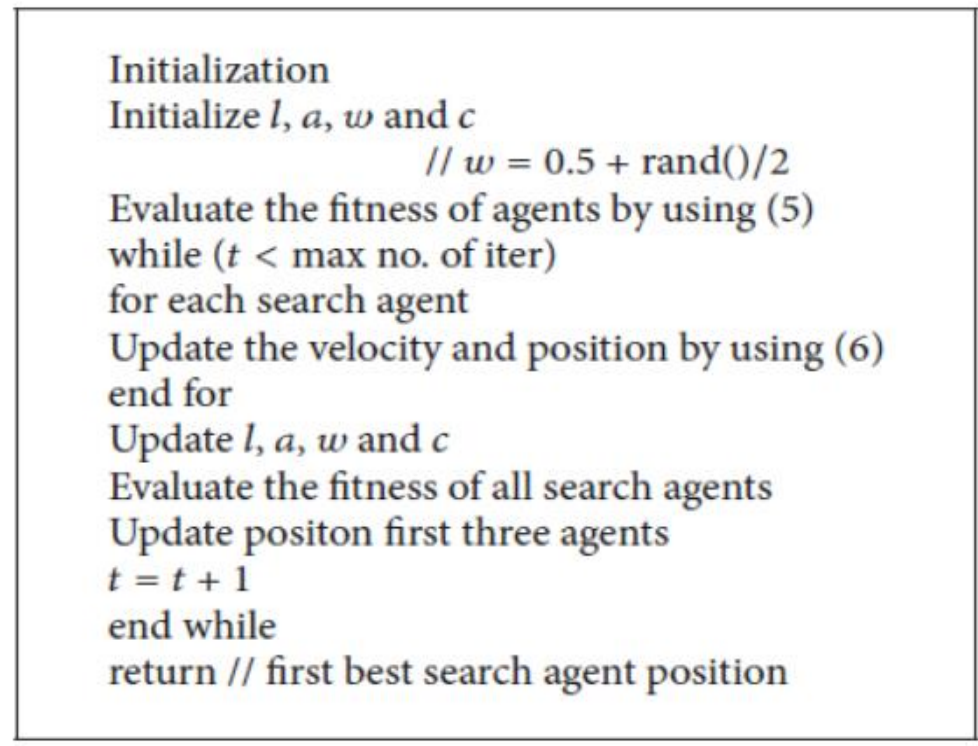

Figure 2. Pseudo Code of the HPSOGWO Algorithm

\section{Formulation of the mathematical model for the problems}

Last few decades have witnessed lot of research in formulation of mixed configuration as pure series or parallel configuration are not enough to design complex system of the real-world engineering problems. The following problems of mixed configuration with both series and parallel structures based on the reliability allocation have been solved using HPSOGWO technique.

In this paper, the two problems considered are complex bridge system and life support system in space capsule. These are nonlinear optimization problems subject to respective constraints of component reliability and system costs.

\subsection{Problem of Complex Bridge system:}

Complex bridge system (Padhye et al., 2009; Pant \& Singh, 2011, Kumar; Pant \& Ram, 2017) has a mixed configuration of series and parallel. The system has a total of 
five components (Fig.3). The system reliability (Rs) and system cost (Cs) of a complex bridge network are given below.

$R_{s}=r_{1} r_{4}+r_{2} r_{5}+r_{2} r_{3} r_{4}+r_{1} r_{3} r_{5}+2 r_{1} r_{2} r_{3} r_{4} r_{5}-r_{1} r_{2} r_{4} r_{5}-r_{1} r_{2} r_{3} r_{4}-r_{2} r_{3} r_{4} r_{5}-$

$r_{1} r_{2} r_{3} r_{5} \quad r_{1} r_{3} r_{4} r_{5}$

$C_{S}=\sum_{i=1}^{5} a_{i} \exp \left[\frac{b}{\left(1-r_{i}\right)}\right]$

The optimization problem in mathematical form is as under:

Minimize $C_{s}$

Subject: $0 \leq r_{i} \leq 1 \quad i=1,2,3,4,5$

$0.99 \leq R_{s} \leq 1$

$a_{i}=1$, and $b_{i}=0.0003, \quad$ for $i=1,2,3,4,5$

where, $R_{i}$ is $i^{\text {th }}$ component's reliability.

\subsection{Problem of space capsule:}

Life support system in space capsule (Anthony, 2006) presented below is composed of 4 components (Fig. 4). This mixed series- parallel system is used for space exploration and the related equations are as follows: (Kumar et al., 2017)

$R_{s}=1-r_{3}\left[\left(1-r_{1}\right)\left(1-r_{4}\right)\right]^{2}-\left(1-r_{3}\right)\left[1-r_{2}\left\{1-\left(1-r_{1}\right)\left(1-r_{4}\right)\right\}\right]^{2}$

$C_{s}=2 K_{1} r_{1}^{\alpha_{1}}+2 K_{2} r_{2}{ }^{\alpha_{2}}+K_{3} r_{3}{ }^{\alpha_{3}}+2 K_{4} r_{4}{ }^{\alpha_{4}}$

where, $K_{1}=100, K_{2}=100, K_{3}=200, K_{4}=150$ and $\alpha_{i}=0.6, i=1,2,3,4,5$

Minimize $C_{s}$

Subject: $0.5 \leq r_{i} \leq 1$

$0.9 \leq R_{s} \leq 1$.

$$
i=1,2,3,4,5
$$

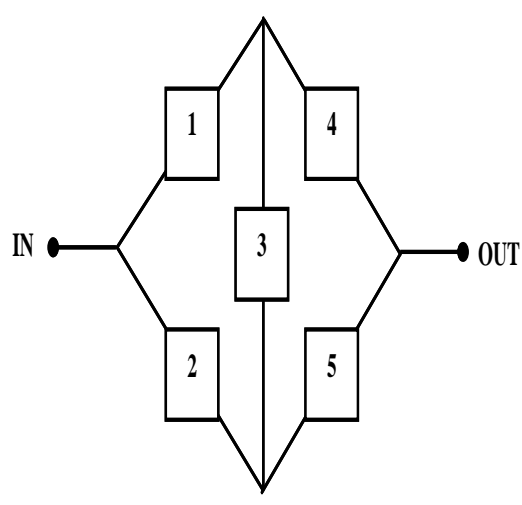

Figure 3. Complex bridge system

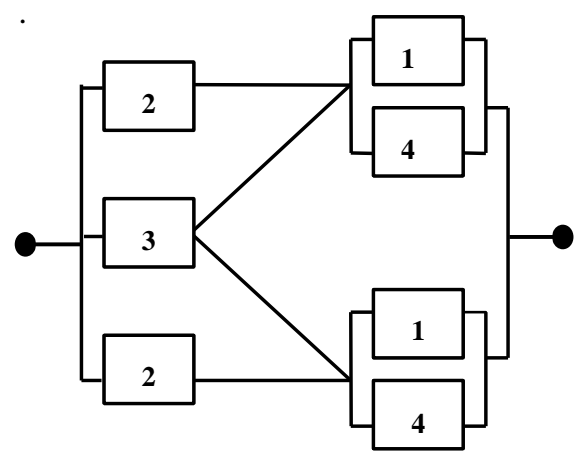

Figure 4. Life support system in a space capsule

\section{Result analysis}

For the above-mentioned problems of reliability allocation, we employed the simplest penalty functions method for constraints handling and the HPSOGWO algorithm has been implemented in MATLAB with number of grey wolves fixed same as GWO and the best results obtained are reported in Table $1 \&$ Table 2. 
Analysis of the results of problem 6.1 Complex Bridge systems shows that with HPSOGWO for 400 iterations and population size of 100 (total number of grey wolf) the number of function evaluations is 40,000 with same reliability as with PSO and GWO individually (Table 1). So, this result is better than that of GWO with regards to number function evaluations (Figure 5).

Table 1: Result comparison for Complex Bridge System

\begin{tabular}{|c|c|c|c|}
\hline \multicolumn{4}{|c|}{ Complex Bridge System } \\
\hline & PSO & GWO & HPSOGWO \\
\hline$r_{1}$ & 0.9348210000 & 0.9341000000 & 0.9308565080 \\
\hline$r_{2}$ & 0.9350280000 & 0.9363500000 & 0.9399944690 \\
\hline$r_{3}$ & 0.7919480000 & 0.7913700000 & 0.8094644730 \\
\hline$r_{4}$ & 0.9350050000 & 0.9338800000 & 0.9354764350 \\
\hline $\mathrm{r}_{5}$ & 0.9347350000 & 0.9356500000 & 0.9313288850 \\
\hline $\begin{array}{c}\text { No. of } \\
\text { Iterations }\end{array}$ & 300 & 300 & 200 \\
\hline$R_{s}$ & 0.99000500000 & 0.99002800000 & 0.99000033494 \\
\hline$C_{s}$ & 5.01991800000 & 5.01990000000 & 5.066228730000 \\
\hline $\mathrm{FE}$ & $1,20,000$ & 9000 & 6000 \\
\hline
\end{tabular}

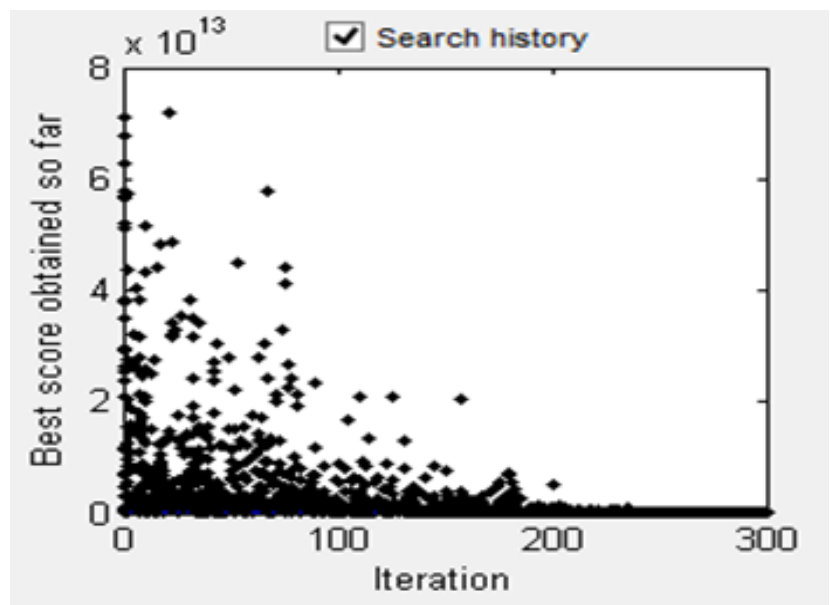

Figure 5. Search history for Problem 6.1

Analysis of the result of problem 6.2 Life support system in a space capsule shows that with HPSOGWO for 200 iterations and population size of just 30 (total number of grey wolf) the result is quite competitive in terms of higher reliability cost minimisation and the number of function evaluations is just 6000 which is far better than the results obtained with PSO and GWO individually (Figure 6). The results obtained are shown in Table 2. 
Optimization of complex system reliability using hybrid Grey Wolf optimizer

Table 2: Result comparison for Life Support System in a Space Capsule

\begin{tabular}{|c|c|c|c|}
\hline \multicolumn{4}{|c|}{ Life Support System in a Space Capsule } \\
\hline & PSO & GWO & HPSOGWO \\
\hline$r_{1}$ & 0.500000000 & 0.500000000 & 0.500000000 \\
\hline$r_{2}$ & 0.838924024 & 0.838920000 & 0.838924024 \\
\hline$r_{3}$ & 0.500000000 & 0.500000000 & 0.500000000 \\
\hline$r_{4}$ & 0.500000000 & 0.500000000 & 0.500000000 \\
\hline $\begin{array}{c}\text { No. of } \\
\text { Iterations }\end{array}$ & 300 & 500 & 400 \\
\hline$R_{s}$ & 0.900000000 & 0.900000000 & 0.900000000 \\
\hline$C_{s}$ & 641.823562000 & 641.823600000 & 641.823562000 \\
\hline $\mathrm{FE}$ & 2040 & 50,000 & 40000 \\
\hline
\end{tabular}

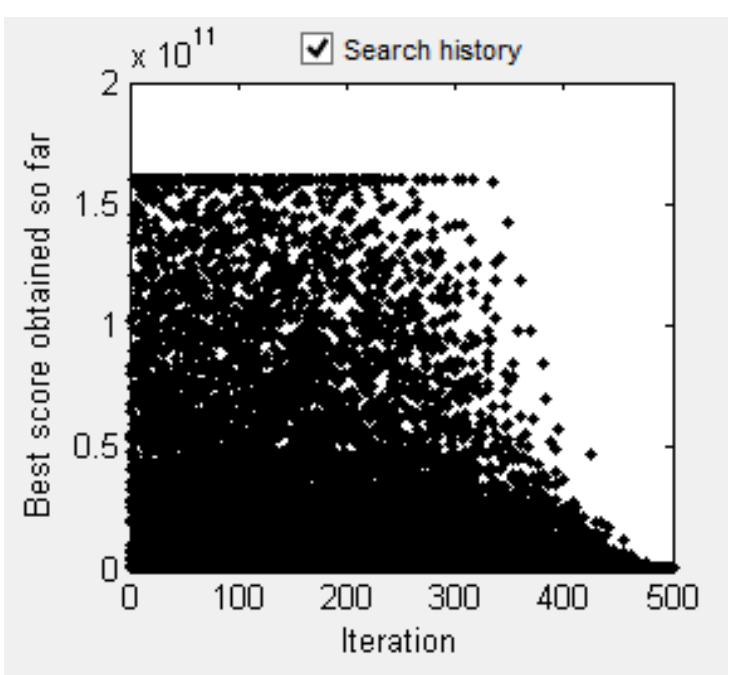

Figure 6. Search history for Problem 6.2

Thus, hybrid of PSO and GWO gives an overall better performance than the individual optimization technique. A comparison of the results clarifies that though results are better than other optimization techniques, but HPSOGWO results better in one or the other form all the previously obtained results.

From the results, it is clear, that HPSOGWO gives comparatively much better results than other metaheuristics like PSO, GWO individually used earlier for such complex reliability allocation problems in terms of the lesser number of function evaluations (Table $1 \&$ Table 2).

\section{Conclusion and further scope}

Nature inspired optimization algorithms have extended their roots in almost all complex optimization problems of modern-day industries. Reliability allocation problems which are usually NP-Hard in nature are one of them. 
Negi et al./Decis. Mak. Appl. Manag. Eng. 4 (2) (2021) 241-256

In this article, a hybrid algorithm named HPSOGWO has been used to solve two complex reliability allocation problems named complex bridge system and life support system in space capsule. HPSOGWO algorithm has proved superior or comparable overall in terms of lesser number of function evaluation as compared to GWO and PSO. Also, the technique can serve the solutions to the various reliability allocation problems (RAPs) and reliability-redundancy allocation problem (RRAPs) with the use of a proper penalty function.

As further scope, the decision makers can decide the allocation of the desired reliability of the components as well as the whole complex system which can be optimized using HPSOGWO. Together with this the repair and maintenance of the components also can be a part of decision as the reliability of the whole system can be managed better to get competitive results with the HPSOGWO technique. Currently, the authors are working on numerous improvements related to the benchmark problems in reliability allocation problems (RAPs) and reliability-redundancy allocation problem (RRAPs).

Author Contributions: Each author has participated and contributed sufficiently to take public responsibility for appropriate portions of the content.

Funding: This research received no external funding.

Declaration of Conflicting Interests: The Authors have no conflict of interests.

\section{References}

Ab Rashid, M.F.F. (2017). A hybrid Ant-Wolf Algorithm to optimize assembly sequence planning problem. Assembly Automation, 37(2), 238-248.

Abd-Elazim, S.M. \& Ali, E.S. (2015). A hybrid particles swarm optimization and bacterial foraging for power system stability enhancement. Complexity, 21(2), 245255.

Abdullah, J. M. \& Ahmed, T. (2019). Fitness Dependent Optimizer: Inspired by the Bee Swarming Reproductive Process. IEEE Access, 7, 43473-43486.

Ahmed, A., Esmin, A. \& Matwin, S. (2013). HPSOM: a hybrid particle swarm optimization algorithm with genetic mutation. International Journal of Innovative Computing, Information and Control, 9(5), 1919-1934.

Atiqullah, M. M., \& Rao, S.S. (1993). Reliability optimization of communication networks using simulated annealing. Microelectronics Reliability, 33, 1303-1319.

Coelho, L.S. (2009). An efficient particle swarm approach for mixed integer programming problem in reliability-redundancy optimization applications. Reliability Engineering and System Safety, 94 (4), 830-837.

Deep, K., \& Deepti. D. (2009). Reliability Optimization of Complex Systems through CSOMGA. Journal of Information and Computing Science, 4, 163-172.

Dorigo, M., \& Gambardella, L.M. (1997). Ant colony system: A cooperative learning approach to the traveling salesman problem. IEEE Trans Evol Comput, 1(1), 53-66. 
Optimization of complex system reliability using hybrid Grey Wolf optimizer

Eberhart, R. \& Kennedy, J. (1995). A new optimizer using particle swarm theory. MHS'95. In Proceedings of the Sixth International Symposium on Micro Machine and Human Science, 39-43, doi: 10.1109/MHS.1995.494215.

Eiben, A. E. \& Schippers, C.A. (1998). On evolutionary exploration and exploitation. Fundamental Informatics, 35(1-4), 35-50.

Fouad, M.M, Hafez, A.I., Hassanien, A.E. \& Snasel, V. (2015). Grey wolves optimizerbased localization approach in WSNs. In: 11th international computer engineering conference (ICENCO). IEEE, 256-260.

Hassan, B.A., \& Rashid, T.A. (2021). Evolutionary clustering algorithm (ECA). Neural Computing and Applications; https://doi.org/10.1007/s00521-020-05649-1

Hikita, M., Nakagawa, Y., Nakashima, K., \& Narihisa, H. (1992). Reliability optimization of systems by a surrogate-constraints algorithm. IEEE Transactions on Reliability, 41, 473-480.

Hikita, M., Nakagawa, Y., Nakashima, K., \& Yamato, K. (1986). Application of the surrogate constraints algorithm to optimal reliability design of systems. Microelectronics and reliability,26, 35-38.

Holden, N., \& Freitas, A.A. (2008). A hybrid PSO/ACO algorithm for discovering classification rules in data mining. Journal of Artificial Evolution and Applications. Article ID 316145, 1-11.

$\mathrm{Hu}, \mathrm{X}$., \& Eberhart, R. (2002), Adaptive particle swarm optimization: Detection and response to dynamic systems. In Congress on Evolutionary Computation, 2, 16661670.

Ibrahim, G.J., Rashid, T. A., \& Akinsolu, M. 0. (2020). An energy efficient service composition mechanism using a hybrid meta-heuristic algorithm in a mobile cloud environment. Journal of parallel and distributed computing, 143.

Jayabarathi, T., Raghunathan, T., Adarsh, B.R., \& Suganthan, P. N. (2016). Economic dispatch using hybrid grey wolf optimizer. Energy, 111, 630-641.

Kennedy, J., \& Eberhart, R. (1997). A discrete binary version of the particle swarm algorithm, in IEEE International Conference on Systems, Man, and Cybernetics, Computational Cybernetics and Simulation, 5, 4104-4108.

Kishor, A., Yadav, S. P., \& Kumar, S. (2007). Application of a Multi-objective Genetic Algorithm to solve Reliability Optimization Problem, In International Conference on Computational Intelligence and Multimedia Applications, 458-462.

Kishor, A., Yadav, S. P., \& Kumar, S. (2009). A Multi-objective Genetic Algorithm for Reliability Optimization Problem. International Journal of Performability Engineering, 5, 227-234.

Kumar, A., Pant, S., \& Ram, M. (2017). System Reliability Optimization Using Grey Wolf Optimizer Algorithm. Quality and Reliability Engineering International, 33(7), 1327 1335.

Kumar, A., Pant, S., \& Ram, M. (2019a). Grey wolf-optimizer approach to the reliabilitycost optimization of residual heat removal system of a nuclear power plant safety system. Quality and Reliability Engineering international, 35 (7), 2228-2239. 
Kumar, A., Pant, S., \& Ram, M. (2019b). Multi-objective grey wolf optimizer approach to the reliability-cost optimization of life support system in space capsule. International Journal of System Assurance Engineering and management, 10 (2), 276284.

Kumar, A., Pant, S., \& Singh, S. B. (2016). Reliability Optimization of Complex System by Using Cuckoos Search algorithm, Mathematical Concepts and Applications in Mechanical Engineering and Mechatronics, IGI Global, 95-112

Kuo, W., \& Prasad, V.R. (2000). An annotated overview of system-reliability optimization. IEEE Transactions on Reliability, 49, 176-187.

Li, L., Xue, B., Niu, B., Tan, L., \& Wang, J. (2008). A novel PSO-DE based hybrid algorithm for global optimization in Advanced Intelligent Computing Theories and Applications: With Aspects of Artificial Intelligence, vol. 5227 of Lecture Notes in Computer Science, 785-793, Springer, Berlin, Germany.

Majety, S. R. V., Dawande, M., \& Rajgopal, J.(1999). Optimal reliability allocation with discrete cost-reliability data for components. Operations Research, 47, 899-906.

Mirjalili, S. M., \& Hashim, S. Z .M. (2010). A new hybrid PSOGSA algorithm for function optimization, in Proceedings of the International Conference on Computer and Information Application (ICCIA '10), pp. 374-377, Tianjin, China.

Mirjalili, S., Mirjalili, S. M., \& Lewis, A. (2014). Grey wolf optimizer. Adv Eng. Soft, 69, 46-61.

Mirjalili, S., Saremi, S., Mirjalili, S. M., \& Coelho, L. S. (2016). Multi-objective grey wolf optimizer: a novel algorithm for multi-criterion optimization. Expert Sys App, 47, 106119.

Misra, K. B., \& Sharma, U. (1991a). An efficient approach for multiple criteria redundancy optimization problems. Microelectronics Reliability, 31, 303-321.

Misra, K. B., \& Sharma, U. (1991b). Multicriteria optimization for combined reliability and redundancy allocation in systems employing mixed redundancies. Microelectronics Reliability, 31, 323-335.

Mohammed, H. M., Abdul, Z. K., Rashid, T. A., Alsadoon, A., \& Bacanin, N. (2021). A new K-means grey wolf algorithm for engineering, World Journal of Engineering ISSN: $1708-5284$

Mohammed, H. M., Umar, S. U., Tarik A., \& Rashid, A. (2019). Systematic and MetaAnalysis Survey of Whale Optimization Algorithm. Computational Intelligence and Neuroscience. https://doi.org/10.1155/2019/8718571.

Mohammed, H., \& Rashid, T. (2020). A novel hybrid GWO with WOA for global numerical optimization and solving pressure vessel design. Neural Computing and Applications, 32, 14701-14718.

Mohan, C., \& Shanker. K. (1987). Reliability optimization of complex systems using random search technique. Microelectronics Reliability, 28, 513-518.

Mosavi, M, R., Khishe, M., \& Ghamgosar, A. (2016). Classification of sonar data set using neural network trained by grey wolf optimization. Neural Net World, 26(4), 393. 
Muhammed, D.A., Saeed, S.A.M., \& Rashid, T.A. (2020). Improved Fitness-Dependent Optimizer Algorithm. IEEE, 8.

Mukherjee, A., Barma, P.S., Dutta, J., Panigrahi, G., Kar, S., \& Maiti, M. (2021). A multiobjective antlion optimizer for the ring tree problem with secondary sub-depots, https://link.springer.com/article/10.1007/s12351-021-00623-8

Negi, G., Kumar, A., Pant, S., \& Ram, M. (2020). GWO: a review and applications, International Journal of System Assurance Engineering and management, https://doi.org/10.1007/s13198-020-00995-8.

Padhye, N., Branke, J. \& Mostaghim, S. (2009). Empirical comparison of MOPSO methods-guide selection and diversity preservation, in IEEE Congress on Evolutionary Computation, 2516-2523.

Pant, S. \& Singh, S.B. (2011). Particle Swarm Optimization to Reliability Optimization in Complex System, In the proceeding of IEEE Int. Conf. on Quality and Reliability, Bangkok, Thailand, 211-215.

Pant, S, Anand, D., Kishor, A., \& Singh, S.B. (2015). A Particle Swarm Algorithm for Optimization of Complex System Reliability. International Journal of Performability Engineering, 11(1), 33-42

Pant, S., Kumar, A., \& Ram, M. (2019). Solution of Nonlinear Systems of Equations via Metaheuristics. International Journal of Mathematical, Engineering and Management Sciences, 4 (5), 1108-1126.

Pant, S., Kumar, A. \& Ram, M. (2017). Flower Pollination Algorithm Development: A State of Art Review. International Journal of System Assurance Engineering and Management, Springer, 8 (2), 1858-1866.

Pham, H., Pham, H. K, \& Amari, S. V. (1995). A general model for evaluating the reliability of telecommunications systems. Commun Reliab Maintain Support-Int J; 2:4-13.

Rahman, C.M. \& Rashid, T. A, (2020). Learner performance-based behaviour algorithm (LPB) https://doi.org/10.1016/j.eij.2020.08.003

Ramírez-Rosado, I. J. \& Bernal-Agustín, J. L. (2001). Reliability and costs optimization for distribution networks expansion using an evolutionary algorithm. IEEE Transactions on Power Systems, 16, 111-118.

Rashid, T. A, Abbas, D. K. \& Turel, Y.K. (2019). A multi hidden recurrent neural network with modified grey wolf optimizer. https://doi.org/10.1371/journal.pone.0213237

Sakawa, M. (1978). Multi-objective reliability and redundancy optimization of a seriesparallel system by the Surrogate Worth Trade-off method. Microelectronics and Reliability, 17, 465-467.

Salazar, D., Rocco, C. M., \& Galván, B. J. (2006). Optimization of constrained multipleobjective reliability problems using evolutionary algorithms. Reliability Engineering \& System Safety, 91, 1057-1070.

Shelokar, P. S, Jayaraman, V. K., \& Kulkarni, B. D. (2002). Ant algorithm for single and multi-objective reliability optimization problems. Quality and Reliability Engineering International, 18, 497-514. 
Negi et al./Decis. Mak. Appl. Manag. Eng. 4 (2) (2021) 241-256

Singh, N. \& Singh, S. B. (2017). Hybrid algorithm of Particle swarm optimization and grey wolf optimizer for improving convergence performance. Journal of Applied Mathematics, Article ID 20304889, 1-16.

Tawhid, M. A. \& Ali, A. F. (2017). A Hybrid grey wolf optimizer and genetic algorithm for minimizing potential energy function. Memetic Computing, 9(4), 347-359.

Uniyal, N. Pant, S., \& Kumar, A. (2020). An Overview of Few Nature Inspired Optimization Techniques and Its Reliability Applications. International Journal of Mathematical, Engineering and Management Sciences, 5 (4), 732-743.

Wolpert, D. H., \& Macready, W. G. (1997). No free lunch theorems for optimization. IEEE transactions on Evolutionary computation; 1, 67-82.

Yang, X.S., and Deb, S. (2009) 'Cuckoo search via L'evy flights', Proceedings of World Congress on Nature \& Biologically Inspired Computing (NBIC, India), IEEE Publications, USA, 210-214.

Zha, J. H. Liu, Z., \& Dao, M. T. (2007). Reliability optimization using multi-objective ant colony system approaches, Reliability Engineering \& System Safety. 92, 109-120.

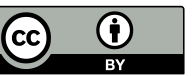

(C) 2021 by the authors. Submitted for possible open access publication under the terms and conditions of the Creative Commons Attribution (CC BY) license (http://creativecommons.org/licenses/by/4.0/). 